\title{
Hyperbolic Icosahedral Tilings by Buckyballs
}

\author{
In memory of Professor Ludwig Danzer
}

R. LÜCK ${ }^{a, *}$ AND D. FRETTLÖH ${ }^{b}$

${ }^{a}$ Weilstetter Weg 16, 70567 Stuttgart, Germany

${ }^{b}$ Bielefeld University, Technical Faculty, PB 100131, 33501 Bielefeld, Germany

\begin{abstract}
Hyperbolic icosahedral tilings by Buckyballs with 4 or 8 Buckyballs sharing a vertex are considered as an example of tilings by Archimedean polyhedra. Representation requires Poincaré balls; colour symmetry may be derived by GAP. Colour symmetries preserving both rotation and reflection symmetry as well as only rotation symmetry are discussed.
\end{abstract}

DOI: 10.12693/APhysPolA.126.524

PACS: 02.20.-a; 61.48.-c; 61.50.Ah; 61.44.Br

\section{Introduction}

Icosahedral polyhedra composed of 20 regular hexagons and 12 regular pentagons are shortly termed Buckyballs, they have the same structure as $\mathrm{C}_{60}$ molecules termed fullerene; the vertices of Buckyballs are occupied by carbon atoms. The structure unit may be abbreviated by (5.6.6). Both, Buckyball and fullerene, are called after the famous architect Buckminster Fuller. A Buckyball consists of 120 fundamental areas, the geometry of the fundamental area is basic to the present investigation. A tiling of Buckyballs - or of fundamental areas - is not possible in Euclidean space. However, one may imagine it in hyperbolic space.

For visualisation of the $3 \mathrm{D}$ hyperbolic tiling the Poincaré disc should be extended to a Poincaré ball. There are other methods of visualisation, as Poincaré half plane, however, it will not be applied in this publication. If possible, two-dimensional intersections of the Poincaré ball may be used, which can result in two-dimensional hyperbolic tilings. Colour indices in Euclidean periodic and quasiperiodic tilings are well known [1, 2]. Colour indices of hyperbolic tilings have been discussed very recently [3-7]. Usually colour symmetries correspond to certain subgroups of the symmetry group. GAP [8] is tailored to computing subgroups of finitely presented groups; it is a system for computational discrete algebra with particular emphasis on computational group theory. The result of this program is the numbers of colour indices and their multiplicity. The program requires as input data the reflection symmetries [9] of the fundamental area. Therefore the fundamental area of the Buckyball and its relation with the hyperbolic tiling must be carefully analysed.

The fundamental area of Buckyballs is 1/120 of a single Buckyball according to icosahedral symmetry (Fig. 1). This is composed of five facets, which may be termed $A, B, C, D$, and $E$.

*corresponding author; e-mail: r.v.lueck@web.de

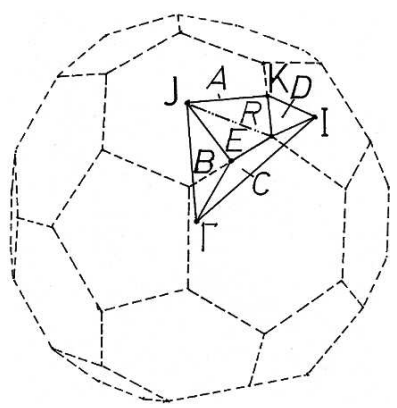

Fig. 1. The fundamental area of Buckyballs is $1 / 120$ of a single Buckyball according to the icosahedral symmetry. It is composed of five facets $A, B, C, D$, and $E$.

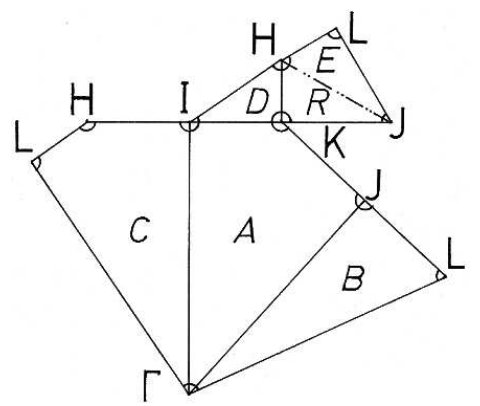

Fig. 2. Devolution of the fundamental area of a Buckyball. Labels $A, B, C, D, E$, or $R$ enter the GAP procedure.

Figure 2 represents a devolution of the fundamental area. There are three quadrangles $(A, C, E)$ and two triangles $(B, D)$ and six vertices: the centre of the Buckyball $(\Gamma)$, the centre of a pentagon $(\mathrm{I})$, the centre of a hexagon $(\mathrm{J})$, a vertex of the Buckyball $(\mathrm{H})$, two centres of edges; there is one centre of an edge sharing two hexagons (L) and another centre on an edge sharing a hexagon and a pentagon $(\mathrm{K})$. We will consider the symmetry of the fundamental area using the known example of four and a novel example with eight Buckyballs sharing a vertex, the authors are not aware of any reference of this tiling. 


\section{Four Buckyballs sharing a vertex}

Four Buckyballs sharing a vertex may be abbreviated as $(5.6 .6)^{4}$.

The symmetry of the vertex figure cannot be tetrahedral $43 m$ ( 4 stands for a fourfold inversion axis) since threefold axes are missing at the vertices. The symmetry is therefore $42 \mathrm{~m}$ (again, the italic letter stands for an inversion axis). The hexagons of the Buckyballs are not reflection planes in this three-dimensional tiling whereas the pentagons are. Therefore, the fundamental area is not exclusively formed by reflection planes. This implies special conditions for the GAP procedure: $A^{2}=B^{2}=C^{2}=D^{2}=1 . E$ is in $(5.6 .6)^{4}$ not a reflection plane, therefore the rotation $R$ should be applied: $R^{2}=1$. Due to icosahedral symmetry it follows: $(C A)^{5}=(A B)^{3}=(B C)^{2}=1$, due to reflection symmetry of $D$ it follows: $(A D)^{2}=(C D)^{2}=1$, and eventually due to rotation: $(A R B R)=(C R D R)=1$.

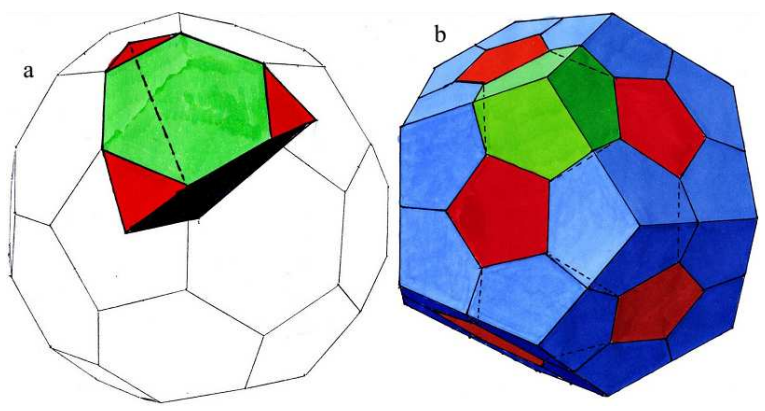

Fig. 3. (a) $1 / 20$ of a Buckyball should be mounted at the hexagon of a central one. (b) A pentagonal dodecahedron is formed by two-colour superstructure. 20 dodecahedra are sharing a vertex resulting in $(5.5 .5)^{20}$.

A symmetric colouring with two colours exists for this tiling. This decoration implies that colour changes at hexagons and does not change at pentagons. From the colouring with two colours an according index 2 superstructure can be deduced. To visualise the superstructure the centres of Buckyballs of the first colour should be transformed to centres of polyhedra of the superstructure, whereas the centres of Buckyballs of the second colour should be transformed to vertices of the superstructure. Therefore, $1 / 20$ of a Buckyball of the second colour should be mounted at the hexagon of a central one, which is coloured with the first colour (Fig. 3a and b). Hexagon at hexagon must be fixed in the correct orientation. A pentagonal dodecahedron is formed. 20 dodecahedra are sharing a vertex resulting in $(5.5 .5)^{20}$. For this tiling the colour symmetries are (up to 77) 57, 59, $60,68,77$, which have been determined by GAP. Accordingly the colour indices 2, 114, 118, 120, 136, 154 can be concluded for $(5.6 .6)^{4}$.

\section{Eight Buckyballs sharing a vertex}

There are restrictions for gluing Buckyballs to a tiling. Not only the symmetries must fit, but also the fraction of pentagons and hexagons sharing a vertex must be compatible with the Buckyball: it is $1 / 3: 2 / 3$. Therefore, a tiling with 20 Buckyballs $(5.6 .6)^{20}$ sharing a vertex with icosahedral symmetry can be ruled out. We tried to design 8 Buckyballs sharing a vertex. The symmetry of the vertex is tetragonal: $4 / \mathrm{mmm}$ (not cubic $m 3 m)$. The hexagon must be adjusted by distortion, but with conservation of symmetry of threefold rotation. Therefore we write $6^{*}$. The tiling may be abbreviated as $\left(5.6^{*} \cdot 6^{*}\right)^{8}$. The following relations were deduced for $\left(5.6^{*} .6^{*}\right)^{8}: A^{2}=B^{2}=C^{2}=D^{2}=E^{2}=1 ;(C A)^{5}=$ $(A B)^{3}=(B C)^{2}=1 ;(A D)^{2}=(C D)^{2}=(B E)^{2}=1$; $(A E)^{2}=(C E)^{4}=(D E)^{2}=1$. Using these relations, GAP yielded in the following colour symmetries preserving both rotation and reflection symmetry (up to $k=16$ ) for $\left(5.6^{*} \cdot 6^{*}\right)^{8}$ (Table I).

TABLE I

The list of the first possible $k$ values and their multiplicities for perfect colourings of the tiling with eight Buckyballs sharing a vertex.

\begin{tabular}{c|c|c|c|c|c|c|c|c}
\hline \hline$k$ & 1 & 2 & 4 & 7 & 12 & 13 & 14 & 16 \\
\hline multiplicity & 1 & 3 & 1 & 1 & 1 & 8 & 15 & 2
\end{tabular}

TABLE II

The list of the first possible $k$ values and their multiplicities for perfect colourings with inclusion of colour symmetries which preserve only rotation symmetry of the tiling with eight Buckyballs sharing a vertex.

\begin{tabular}{c|c|c|c|c|c|c|c|c}
\hline \hline$k$ & 1 & 2 & 4 & 7 & 12 & 13 & 14 & 16 \\
\hline multiplicity & 1 & 3 & 1 & 1 & 5 & 10 & 20 & 2
\end{tabular}

The corresponding table with inclusion of colour symmetries which preserve only rotation symmetry (up to $k=16$ ) for $\left(5.6^{*} \cdot 6^{*}\right)^{8}$ is listed in Table II.

Only a few examples will be discussed.

\subsection{Two and four colours in $\left(5.6^{*} \cdot 6^{*}\right)^{8}$}

Three versions of a two-colour decoration can be easily understood. Colour may change (i) at pentagons, (ii) at hexagons or (iii) at both pentagons and hexagons. If the colours are characterised by two binary digits, one digit may change at pentagons and the other at hexagons. In this manner the four-colour decoration with $(0,0),(0,1)$, $(1,0)$ and $(1,1)$ may be easily developed.

\subsection{Seven colours in $\left(5.6^{*} \cdot 6^{*}\right)^{8}$}

For the colour index $k=7$, colours will only change at pentagons and not at hexagons. The relationships can be easily understood.

\subsection{Sixteen colours in $\left(5.6^{*} .6^{*}\right)^{8}$}

16 colours can be characterised by 4 binary digits. There are 15 different types of colour change, each one characterised by a set of four binary digits. There is no change of colour at pentagons, change of colour will occur at hexagons resulting in 10 different colours. Change to the remaining 5 different colours will occur at those edges which are between two hexagons. 


\subsection{Six colours in $\left(5.6^{*} \cdot 6^{*}\right)^{8}$ with only rotational colour symmetry}

This type of colour decoration requires colour change at hexagons and no change at pentagons. Five colours are arranged around a Buckyball in chiral manner. For each of the 5 colours there are 4 Buckyballs neighbouring and the centres of these 4 Buckyballs form a tetrahedron. Figure 4 represents 2D intersections perpendicular to the fourfold axis decorated with 6 colours with only rotation colour symmetry. There exists an enantiomorphic pair of colour decoration. This colour decoration is a special case; it produces - strictly spoken - not an enantiomorphic pair of tilings, since the chirality is not constant within the tiling; moreover it changes at pentagons. Nevertheless, two such tilings combined form perfect colour symmetry with 36 colours.
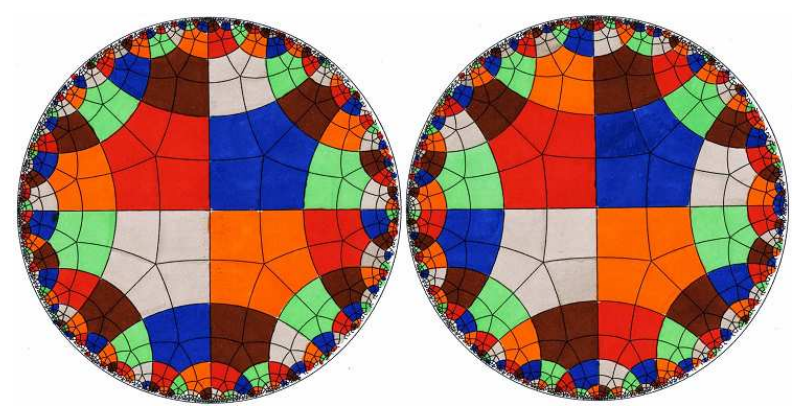

Fig. 4. Intersection of three-dimensional Buckyball tiling $\left(5.6^{*} .6^{*}\right)^{8}$ perpendicular to the fourfold axis forms a two-dimensional hyperbolic pentagon tiling $\left(5^{4}\right)$. This tiling can be coloured with 6 colours in two different manners [3]. The presented six-colour decorations exhibiting only rotational colour symmetry represent intersections of the chiral colour decoration of the three-dimensional tiling.

\section{Conclusions and outlook}

Hyperbolic three-dimensional tilings composed of Archimedean polyhedra can be coloured with perfect colour symmetry as well as tilings composed of (regular) Platonic polyhedra. There are some relations between them due to superstructures.

Visualisation is a challenge.

GAP is an important tool to compute colour indices and their multiplicity.

Other Buckyball tilings with altered hexagons may be investigated. Several vertex configurations are of interest.

\section{Acknowledgments}

This project would not have come to the present result without former or present cooperation with and assistance of Michael Baake, Shelomo I. Ben-Abraham, Manuel Loquias and Max Scheffer.

\section{References}

[1] M. Baake, J. Phys. A, Math. Gen. 30, 2687 (1997).

[2] R. Lück, Philos. Mag. 88, 2049 (2008) and references therein.

[3] D. Frettlöh, Z. Kristallogr. 223, 773 (2008).

[4] R. Lück, D. Frettlöh, Z. Kristallogr. 223, 782 (2008).

[5] M.L.A.N. de las Peñas, R.P. Felix, G.R. Laigo, Z. Kristallogr. 221, 665 (2006).

[6] M.L.A.N. de las Peñas, R.P. Felix, G.R. Laigo, Z. Kristallogr. 225, 313 (2010).

[7] M.L.A.N. de las Peñas, R.P. Felix, B.R. Gozo Jr. G.R. Laigo, Philos. Mag. 91, 2700 (2011).

[8] GAP System for Computational Discrete Algebra, GAP - Groups, Algorithms and Programming.

[9] J. Humphreys, Reflection Groups and Coxeter Groups, series Cambridge Studies in Advanced Mathematics, No. 29, Cambridge University Press, Cambridge 1990; in Cambridge Books Online, Cambridge 2012. 